\title{
Significance of CTNNB1 Mutation in Cellular Process, Prognosis, and Drug Selection in gastric cancer
}

\section{tianqiong Cheng}

Department of Clinical Medicine, Graduate School of Zunyi Medical University xuenong chen ( $\boldsymbol{Q}$ cheng1314yuan@gmail.com ) Affiliated Hospital of Zunyi Medical College https://orcid.org/0000-0003-0759-8570

\section{Research}

Keywords: gastric cancer, CTNNB1 mutation, TCGA, RNA sequencing, bioinformatics analysis

Posted Date: April 28th, 2020

DOI: https://doi.org/10.21203/rs.3.rs-23144/v1

License: (c) (1) This work is licensed under a Creative Commons Attribution 4.0 International License. Read Full License 


\section{Abstract}

Background: Numerous studies have reported that CTNNB1 mutant is a frequently mutated gene found in gastric cancer (GC), making this a valuable biomarker for GC. However, the underling mechanism of CTNNB1 regulating GC remains unknown.

Aim of the study: To identify the significance of the CTNNB1 mutation in the prognosis and drug selection of GC, evaluate important genes and pathways that are involved in the pathogenesis of GC.

Materials and methods: Data sets were downloaded from the Cancer Genome Atlas (TCGA)-STAD. CTNNB1 mutation and drug sensitivity analysis based on GDSC database. CTNNB1 wild group and mutant group analyzed by GSEA enrichment. The differentially expressed genes (DEGs) were identified by LIMMA package for further GO and KEGG analysis. A protein-protein interaction (PPI) network of DEGs was constructed by STRING and visualized by Cytoscape software.

Results: The CTNNB1 mutation is found in $8 \%$ of GC patients. Patients with CTNNB1 mutations had better prognosis on overall survival and disease-free survival. GC cells with CTNNB1 mutation are sensitive to Nutlin-3a. In total 727 genes were identified as DEGs. GO and KEGG analysis suggested that DEGs were significantly enriched in categories associated with cell differentiation and immune response, digestion related pathways and multiple signal transduction related pathways. Top three significant modules of genes in the PPI network were identified and analyzed.

Conclusions: Our results indicated that multiple genes and pathways may play a key role in GC patients with CTNNB1 mutation, implicated these factors as possible targets for future therapeutic strategies in patients with GC.

\section{Introduction}

Gastric cancer (GC) is the sixth most common cancer in the world [1]. According to statistics from the International Cancer Research Agency, the number of newly diagnosed and fatal cases of gastric cancer in 2018 were 1.03 million and 780,000 respectively [1]. The traditional treatment methods for GC include gastrectomy and chemoradiation, but the recurrence rate and mortality are high, and the 5-year overall survival (OS) is less than $25 \%[2,3]$. Although the current clinical diagnosis and treatment technology for gastric cancer has been continuously improved, it still lacks early, reliable and effective diagnostic and prognostic markers. Therefore, it is of great significance to find biomarkers of gastric cancer prognosis, their roles in gastric cancer, and potential molecular regulatory mechanisms, and to provide clues and ideas for clinical diagnosis, prognostic judgment and new therapeutic targets of gastric cancer.

With the development of modern medicine, cancer diagnosis and treatment have made significant progress in genomics, so that the diagnosis and treatment of gastric cancer has entered a stage of precision and individualization. At present, there are families of ErbB receptors, which are the main molecular signaling pathways of gastric cancer, epidermal growth factor receptor (EGFR) and targeted 
drugs targeting the vascular endothelial growth factor receptor (VEGF) family: trastoma Bezumab, cetuximab, bevacizumab, etc. Analysis of large genomics databases through bioinformatics can screen molecular markers related to the occurrence, development and prognosis of gastric cancer, providing reliable targets for precision medicine at the molecular level.

Mutations of signaling molecules are important factors for cancer development. Catenin beta 1 (CTNNB1 or $\beta$-catenin) interacts with signaling molecules upon stimulation, leading to the transcription of genes related to cell proliferation [4,5]. CTNNB1 signaling is important for revealing cancer mechanisms, meanwhile playing an important role in the phenotypic transition of gastrointestinal cancer $[6,7]$. Telomerase reverse transcriptase gene (TERT) activates Wnt / $\beta$-catenin signaling and the expression of TERT in gastric cancer is related to advanced TNM stage and lymphatic metastasis, suggesting that TERT may be treatment targets for patients with gastric cancer[8]. According to reports, MicroRNA-1225$5 p$ (miR-1225-5p) can inhibit the proliferation and metastasis of gastric cancer by inhibiting the $\beta$-catenin signaling pathway[9].

In this study, we analyzed the RNA sequence (RNA-Seq) dataset of GC and GDSC database to identify key pathways and genes associated with CTNNB1 mutations, and evaluated the significance in drug selection, hopefully it can reveal the potential role of CTNNB1 mutation in prognostic prediction and provide molecular evidence for the diagnosis and treatment of gastric cancer.

\section{Materials And Methods}

\subsection{Data Source}

We downloaded the gastric cancer RNA-Seq dataset and clinical profiles from the Cancer Genome Atlas (TCGA) database (https://portal.gdc.cancer.gov/). Information about patients with CTNNB1 mutant was downloaded from the cBioPortal for Cancer Genomics website (http:// www.cbioportal.org/index.do).

\subsection{Data Mining and Analysis of the GDSC Database}

The Genomics of Drug Sensitivity in Cancer (GDSC) database (www.cancerRxgene.org) is based on queries of specific anticancer drugs or cancer genes and is dedicated to identifying molecular biomarkers for drug sensitivity to facilitate the discovery of new therapeutic biomarkers for cancer therapies[10]. The site focuses on presenting cell line drug sensitivity data and genomic correlations of drug sensitivity, all data can be downloaded. We first searched for compounds that were significantly selective for CTNNB1 mutations. Then we try to narrow the range by checking the sensitivity only in gastric cancer. Volcano maps, elastic nets, scatter maps, and Mann-Whitney-Wilcoxon (MWW) tests were generated and calculated on the GDSC online platform.

\subsection{Gene Set Enrichment Analysis (GSEA)}

We analyzed GSEAv4.0.3 (http://software.broadinstitute.org/gsea/downloads.jsp) to find out the expression levels of differential biological function annotations of gene mRNA and the pathway between 
CTNNB1 mutations and wild-type patients, helping us learned about CTNNB1 mutation affecting various biological functional gene sets of patients with gastric cancer. The number of permutations was set at 5 . The enrichment result meets the $P$-value cutoff 0.05 and false discovery rate (FDR q-val) $<0.25$ is statistically significant.

\subsection{Screening and analysis Differentially Expressed Genes (DEGs)}

The Bioconductor package, "edgeR" ( http:// www.bioconductor.org/packages/release/bioc/html/edgeR.html) was utilized to identify DEGs between patients with CTNNB1 mutations and wild-type gastric cancer. The screening criterion: | Fold Change (FC) $I \geq 2, P$-value and FDR $<0.05$. The DEGs would be used for further analysis. Biological pathway analysis is based on the Kyoto encyclopedia of genes and genomes (KEGG) biological pathway database. This is an important method for studying biological functions from the perspective of complex regulatory networks and performing biological pathway enrichment analysis of gene sets. Genome Ontology (GO) analysis and Kyoto Encyclopedia of Genes and Genomes (KEGG) analysis on the selected differential genes was carried out using R3.6.1 "clusterProfiler" "ggplot2 " "pheatmap" package. The GO and KEGG pathway entries can provide reference information for exploring the biological functions of these differentially expressed genes, especially their role in gastric cancer.

\subsection{PPI network construction}

The PPI network of DEGs obtained from STRING (https://string-db.org) with confidence $>0.7$ as a cut-off criterion $[11,12]$. Then, the data of the PPI network was established using Cytoscape software (version 3.7.2, https://cytoscape.org). The MCODE plug-in in Cytoscape is used to perform modular analysis, and the most important modules are determined based on the MCODE score and node number.

\subsection{Statistical analysis}

mRNA expression level between wild-type and CTNNB1 mutation GC tissue were compared using the $t$ test. Kaplan-Meier survival analysis by log-rank test was used to calculate the clinical outcome between different CTNNB1 groups. FDR in edgeR and GSEA were adjusted using the Benjamini-Hochberg procedure. $P<0.05$ were considered statistically significant. All the statistical analyses were conducted with R 3.6.1.

\section{Results}

\subsection{Patient Characteristics}

We obtained the complete transcriptional expression profiles and clinical information for 380 gastric cancer patients from TCGA cohort. CTNNB1 gene mutation in 33 patients with gastric cancer (8\%), and the rest were CTNNB1 wild type (Fig. 1A). Mutation types included inframe mutation, truncating and missense mutations (Fig. 1B). These data were got from the cBioPortal for Cancer Genomics website. 


\subsection{Clinical Impact of CTNNB1 Mutation in GC Progress and Prognosis}

The clinical characteristics of the two groups of gastric cancer patients are listed in Table 1. We first determined CTNNB1 mRNA expression levels in the wild-type and mutant groups. The results showed that CTNNB1 up-regulated in tumor tissues of patients with mutant gastric cancer (Fig. 2A). Analysis of the relationship between CTNNB1 status and disease prognosis showed that patients with CTNNB1 mutations had better prognosis on overall survival (Fig. 2B) and disease-free survival (Fig. 2C), which indicated that CTNNB1 mutations may be helpful for the treatment of patients with gastric cancer. 
Table 1

Clinical characteristics of GC patients and

CTNNB1 status in TCGA

\begin{tabular}{|c|c|c|}
\hline \multirow[t]{2}{*}{ Characteristics } & \multicolumn{2}{|c|}{ CTNNB1 stutas } \\
\hline & Wild type & Mutated \\
\hline Age, years & 65.5 & 63.5 \\
\hline Range & $30-90$ & $35-84$ \\
\hline \multicolumn{3}{|l|}{ Gender } \\
\hline Female & 140 & 17 \\
\hline Male & 243 & 7 \\
\hline \multicolumn{3}{|l|}{ Tumor stage } \\
\hline T1 & 16 & 2 \\
\hline T2 & 78 & 5 \\
\hline T3 & 180 & 10 \\
\hline $\mathrm{T} 4$ & 109 & 7 \\
\hline \multicolumn{3}{|l|}{$\mathrm{N}$ stage } \\
\hline NO & 116 & 12 \\
\hline N1 & 100 & 7 \\
\hline N2 & 78 & 4 \\
\hline N3 & 84 & 1 \\
\hline NX & 5 & 0 \\
\hline \multicolumn{3}{|l|}{ Stage } \\
\hline 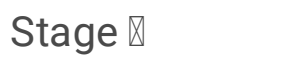 & 49 & 6 \\
\hline 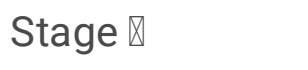 & 120 & 9 \\
\hline 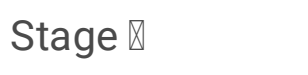 & 174 & 6 \\
\hline Stage $\mathbb{Z}$ & 40 & 3 \\
\hline \multicolumn{3}{|l|}{ Histologic grade } \\
\hline G1 & 9 & 0 \\
\hline G2 & 135 & 5 \\
\hline G3 & 232 & 18 \\
\hline
\end{tabular}




\begin{tabular}{|lll|}
\hline Characteristics & \multicolumn{2}{l|}{ CTNNB1 stutas } \\
\cline { 2 - 3 } & Wild type & Mutated \\
\hline GX & 7 & 1 \\
\hline
\end{tabular}

\subsection{GC Cells With CTNNB1 Mutation Are Sensitive to Nutlin- $3 a$}

In addition to the CTNNB1 mutations on disease progression, we also investigated the role of CTNNB1 mutations in the treatment of GC patients. We studied the GDSC database to find if patients with CTNNB1 mutations have potential selective compounds. The results show that Nutlin-3a has significant selectivity for CTNNB1 mutations in various cancer types (Fig. 2D), making it a potential compound for patients with CTNNB1 mutations. We then studied the tissue specificity of Nutlin-3a and found it was sensitive to gastric cancers with CTNNB1 mutations in the GDSC database (Fig. 2E, F). Nutlin-3a inhibits the p53 / MDM2 interaction and conferred selective inhibition in GC cells with CTNNB1 mutations, making it a potential individualized compound for such GC patients.

\subsection{GSEA}

To further investigate the mechanism of CTNNB1 mutations in GC progression, prognosis, and drug selection, we analyzed the effects of CTNNB1 mutations on cellular processes. First, a variety of biologically functional gene sets were analyzed using the GSEA method. As shown in Fig. 3, the results showed that cell cycle, RNA degradation, spliceosome, pyrimidine metabolism, p53 signaling pathway were significantly enriched. These suggest that CTNNB1 mutations may promote GC progression by affecting multiple factors.

\subsection{Functional Enrichment Analysis of DEGs}

The RNA-Seq data set from 22 patients with CTNNB1 mutations and 353 CTNNB1 wild-type gastric cancer patients were used for DEG screening. Based on $\mid$ fold change $(F C) \mid \geq 2.0$ and $P<0.05$ criteria,a total of 727 genes were identified as DEGs. The heatmap of DEGs shows the first 40 genes (Fig. 4A). The volcano plot of the DEGs is shown in Fig. 4B. To better understand the role of DEGs, KEGG and GO analyses were carried out. The GO analysis of DEGs (Fig. 4C) suggested significant enrichment in cornification, digestion, antimicrobial humoral response, keratinization, keratinocyte differentiation, epidermis development, humoral immune response, disruption of cells of other organism, antimicrobial humoral immune response mediated by antimicrobial peptide. Furthermore, in the KEGG pathway analysis (Fig. 4D), DEGs were significantly enriched in pancreatic secretion, protein digestion and absorption, neuroactive ligand-receptor interaction, maturity onset diabetes of the young, fat digestion and absorption, PPAR signaling pathway, bile secretion, cAMP signaling pathway.

\subsection{PPI network analysis and key target screening}


To better investigate the interaction and hub genes of the identified DEGs, we obtained protein-protein interaction (PPI) networks using the STRING tool. The network was included 512 nodes and 704 edges. Top 30 hub genes were identified by degree (Fig. 5A). Top 10 hub genes included ALB, AHSG, APOA1, APOA2, PENK, ORM2, AFP, APOA5, CHGB, F2. Top three significant modules of genes in the PPI network were identified by the MCODE plugin in Cytoscape for further $G O$ and KEGG pathway enrichment analysis. The first module (Fig. 5B) contains 15 nodes and 210 edges with a MCODE score of 15; the second module (Fig. 5C) contains 24 nodes and 136 edges with a MCODE score of 11.83; the third module (Fig. 5D) contains 22 nodes and 87 edges with a MCODE score of 8.29. The enrichment analysis showed that modules $1-3$ (Table 2) were mainly related to $G$ protein-coupled receptor binding, secondmessenger-mediated signaling, neuropeptide signaling pathway, G protein-coupled receptor signaling pathway, coupled to cyclic nucleotide second messenger, endoplasmic reticulum lumen,post-translational protein modification, epidermal cell differentiation.

Table 2. GO and KEGG analyses of module 1-3.

GO and KEGG analyses of module 1.

\begin{tabular}{llll}
\hline ID & Description & p.adjust & Count \\
\hline GO:0005788 & endoplasmic reticulum lumen & $2.32 \mathrm{E}-25$ & 15 \\
GO:0043687 & post-translational protein modification & $1.26 \mathrm{E}-24$ & 15 \\
GO:0045807 & positive regulation of endocytosis & $1.70 \mathrm{E}-07$ & 6 \\
GO:0004857 & enzyme inhibitor activity & $5.99 \mathrm{E}-06$ & 6 \\
GO:0051346 & negative regulation of hydrolase activity & $1.29 \mathrm{E}-05$ & 6 \\
\hline
\end{tabular}

GO and KEGG analyses of module 2 .

\begin{tabular}{llll}
\hline ID Description p.adjust Count & & \\
\hline GO:0070268 & cornification & $3.57 \mathrm{E}-54$ & 24 \\
GO:0031424 & keratinization & $1.19 \mathrm{E}-46$ & 24 \\
GO:0030216 & keratinocyte differentiation & $1.85 \mathrm{E}-43$ & 24 \\
GO:0009913 & epidermal cell differentiation & $7.49 \mathrm{E}-42$ & 24 \\
GO:0043588 & skin development & $2.94 \mathrm{E}-40$ & 24 \\
GO:0008544 & epidermis development & $3.03 \mathrm{E}-39$ & 24 \\
\hline
\end{tabular}


GO and KEGG analyses of module 3.

\begin{tabular}{llll}
\hline ID & Description & p.adjust & Count \\
\hline GO:0048018 & receptor ligand activity & $1.24 \mathrm{E}-10$ & 11 \\
GO:0008528 & G protein-coupled peptide receptor activity & $9.96 \mathrm{E}-12$ & 9 \\
GO:0001653 & peptide receptor activity & $9.96 \mathrm{E}-12$ & 9 \\
GO:0019932 & second-messenger-mediated signaling & $3.46 \mathrm{E}-08$ & 9 \\
GO:0005179 & hormone activity & $1.01 \mathrm{E}-10$ & 8 \\
GO:0019933 & cAMP-mediated signaling & $1.93 \mathrm{E}-09$ & 8 \\
GO:0019935 & cyclic-nucleotide-mediated signaling & $4.92 \mathrm{E}-09$ & 8 \\
\hline
\end{tabular}

\section{Discussion}

CTNNB1 is a protein-coding gene that encodes a protein that is a key downstream component of the canonical Wnt signaling pathway. The Wnt / $\beta$-catenin signaling pathway plays an important role in tumors by regulating events such as cell survival, proliferation and differentiation. CTNNB1 gene mutation can cause abnormal expression of $\beta$-catenin protein in the cell, resulting in abnormal activation of Wnt / $\beta$-catenin signaling pathway. Aberrant activation of the classical Wnt signaling pathway has been confirmed in various human malignancies including gastric cancer [13-16]. Mutations in APC and CTNNB1 were prevalent in gastric carcinoma [17], which is responsible for gastrointestinal tumorigenesis. In another study, CTNNB1 mutations also were detected in all gastrointestinal tumor samples[18]. Betacatenin is a multifunctional product of the CTNNB1 gene [19], which mediates cell adhesion and signal transduction. Overexpression of $\beta$ catenin encoded by CTNNB1 gene might play important role in tumorigenesis and tumor progression[20]. Therefore, blocking the Wnt signaling pathway may be a promising strategy for cancer treatment.

In this study, we intend to identify the clinical significance of CTNNB1 mutations in GC progression, prognosis, and drug selection, to providing new ideas for the diagnosis, treatment, and prognosis of gastric cancer. We found that about $8 \%$ of 380 patients carry CTNNB1 mutations, including inframe mutation, truncating and missense mutations. Clinical analysis shows that the overall survival and disease-free survival of gastric cancer patients with CTNNB1 mutation is significantly improved.

Detection of CTNNB1 mutation can help clinicians to judge the prognosis of gastric cancer patients and effectively choose a better personalized treatment strategy. In addition, the data from GDSC showed preliminary evidence that Nutlin-3a displayed sensitivity for GC with CTNNB1 mutation, which provides more evidence for the application of specific anti-tumor drugs to such patients and provides a foundation for further research. The compound could be used as a targeted drug for GC patients with CTNNB1 mutation.

To further investigate the mechanism of CTNNB1 mutations in GC progression, prognosis and drug selection, as well as identifying key pathways, we analyzed the GC RNA-Seq data set downloaded from 
TCGA. GSEA analysis in this study showed that CTNNB1 mutations were significantly associated with cell cycle, RNA degradation, spliceosome, pyrimidine metabolism, and p53 signaling pathway. P53 is an important tumor suppressor gene, which combines and activates the promoter of downstream target genes, promotes gene transcription, and participates in important processes such as tumor proliferation, apoptosis, and DNA damage $[21,22]$. There are various ways to regulate $\mathrm{p} 53$, thereby regulating tumor cell senescence, which has potential clinical application prospects [23].

A total of 727 DEGs were identified in the RNA-Seq dataset from 22 CTNNB1 mutant patients and 353 CTNNB1 wild-type gastric cancer patients. Functional analysis of DEGs showed that differentially expressed genes in CTNNB1 mutant gastric cancer patients were mainly involved in the antimicrobial humoral response, humoral immune response. In addition, in KEGG pathway analysis, DEGs were significantly enriched in pancreas secretion, protein digestion and absorption, PPAR signaling pathway, bile secretion, and CAMP signaling pathway. The above indicates that CTNNB1 mutation may be involved in the occurrence and development of gastric cancer. The occurrence of gastric cancer is not mediated by a single factor, but a multi-step process involving many complex cell signaling pathways.

In the PPI network analysis, we identified the top 30 genes with the highest degree of interaction, among which ALB had the highest degree of nodes with 32. This gene encodes the most abundant protein in human blood. Gene Ontology (GO) annotations related to this gene include enzyme binding and chaperone binding. An important paralog of this gene is AFP. The KEGG and GO enrichment analysis of the three most important modules in the PPI network showed that modules 1-3 were mainly related to $G$ protein-coupled receptor binding, second-messenger-mediated signaling, neuropeptide signaling pathway, $G$ protein-coupled receptor signaling pathway, coupled to cyclic nucleotide second messenger, endoplasmic reticulum lumen,post-translational protein modification, epidermal cell differentiation. It indicates that gastric cancer cells with CTNNB1 mutation are more active in cell differentiation and cell signal transmission.

Our study is only a preliminary exploration of whether CTNNB1 mutations in GC affect disease progression, prognosis, and drug selection. The mechanism of CTNNB1 mutation in GC needs further verification. At present, it is still an important research direction to find new mutant genes related to GC in the research of GC, and to further explore the role of related cell signaling pathways in the occurrence and progression of GC. It is believed that in the near future, with the advancement of molecular biology technology and the development of the etiology and mechanism of GC, we will be able to develop new therapeutic methods for GC through molecular targets in gastric cancer-related cell signaling pathways.

In conclusion, With this study, we identified the main pathways and genes associated with the CTNNB1 mutation in GC, implicated these factors as possible targets for future therapeutic strategies in patients with GC.

\section{Abbreviations}

GC 
gastric cancer

TCGA

the Cancer Genome Atlas

DEGs

differentially expressed genes

PPI

protein-protein interaction

EGFR

epidermal growth factor receptor

VEGF

vascular endothelial growth factor

TERT

Telomerase reverse transcriptase gene

RNA-Seq

RNA sequence

GDSC

Genomics of Drug Sensitivity in Cancer

MWW

Mann-Whitney-Wilcoxon tests

GSEA

Gene Set Enrichment Analysis

\section{Declarations}

Ethics approval and consent to participate[Not applicable.

Consent for publication $\square A \mid l$ the authors have read and approved the final version of manuscript and give consent.

Competing interests $\square$ No competing interests.

\section{No funding was received}

Authors' contributions[Tianqiong Cheng designed the study, carried out most of the studies, performed the statistical analysis, and drafted the manuscript. Xuenong Chen critically revised the manuscript, provided professional advice.

Acknowledgements $\square$ None.

Availability of data and material[Publicly available datasets were analyzed in this study. This data can be found here: https://portal.gdc.cancer.gov/. 


\section{References}

1. Bray F, Ferlay J, Soerjomataram I, Siegel RL, Torre LA, Jemal A. Global cancer statistics 2018: GLOBOCAN estimates of incidence and mortality worldwide for 36 cancers in 185 countries. Cancer J Clin. 2018;68:394-424.

2. Oh HJ, Lim C-H, Yoon B-H, Yoon SB, Baeg MK, Kim WC, Cho YK, Park JM, Choi M-G, Yoo HM, et al: Fracture after gastrectomy for gastric cancer: A long-term follow-up observational study. European journal of cancer (Oxford, England: 1990) 2017, 72:28-36.

3. van der Post RS, Oliveira C, Guilford P, Carneiro F. Hereditary gastric cancer: what's new? Update 2013-2018. Familial cancer. 2019;18:363-7.

4. Tanabe S, Aoyagi K, Yokozaki H, Sasaki H. Regulation of CTNNB1 signaling in gastric cancer and stem cells. World J Gastrointest Oncol. 2016;8:592-8.

5. Chi J, Zhang H, Hu J, Song Y, Li J, Wang L, Wang Z. AGR3 promotes the stemness of colorectal cancer via modulating Wnt/ $\beta$-catenin signalling. Cellular signalling. 2020;65:109419.

6. Clevers H, Nusse R. Wnt/ $\beta$-catenin signaling and disease. Cell. 2012;149:1192-205.

7. Song X, Xin N, Wang W, Zhao C. Wnt/ $\beta$-catenin, an oncogenic pathway targeted by H. pylori in gastric carcinogenesis. Oncotarget. 2015;6:35579-88.

8. Tang B, Xie R, Qin Y, Xiao Y-F, Yong X, Zheng L, Dong H, Yang S-M. Human telomerase reverse transcriptase (hTERT) promotes gastric cancer invasion through cooperating with c-Myc to upregulate heparanase expression. Oncotarget. 2016;7:11364-79.

9. Zheng H, Zhang F, Lin X, Huang C, Zhang Y, Li Y, Lin J, Chen W, Lin X. MicroRNA-1225-5p inhibits proliferation and metastasis of gastric carcinoma through repressing insulin receptor substrate-1 and activation of $\beta$-catenin signaling. Oncotarget. 2016;7:4647-63.

10. Yang W, Soares J, Greninger P, Edelman EJ, Lightfoot H, Forbes S, Bindal N, Beare D, Smith JA, Thompson IR, et al. Genomics of Drug Sensitivity in Cancer (GDSC): a resource for therapeutic biomarker discovery in cancer cells. Nucleic acids research. 2013;41:D955-61.

11. Szklarczyk D, Gable AL, Lyon D, Junge A, Wyder S, Huerta-Cepas J, Simonovic M, Doncheva NT, Morris JH, Bork P, et al. STRING v11: protein-protein association networks with increased coverage, supporting functional discovery in genome-wide experimental datasets. Nucleic acids research. 2019;47:D607-13.

12. Szklarczyk D, Franceschini A, Wyder S, Forslund K, Heller D, Huerta-Cepas J, Simonovic M, Roth A, Santos A, Tsafou KP, et al. STRING v10: protein-protein interaction networks, integrated over the tree of life. Nucleic acids research. 2015;43:D447-52.

13. Chang B, Tessneer KL, McManus J, Liu X, Hahn S, Pasula S, Wu H, Song H, Chen Y, Cai X, et al. Epsin is required for Dishevelled stability and Wnt signalling activation in colon cancer development. Nature communications. 2015;6:6380.

14. Xie J, Zhang Y, Hu X, Lv R, Xiao D, Jiang L, Bao Q: Norcantharidin inhibits Wnt signal pathway via promoter demethylation of WIF-1 in human non-small cell lung cancer. Medical oncology 
(Northwood, London, England) 2015, 32:145.

15. Ashihara E, Takada T, Maekawa T. Targeting the canonical Wnt/ß-catenin pathway in hematological malignancies. Cancer Sci. 2015;106:665-71.

16. Fang F, Zhao W-Y, Li R-K, Yang X-M, Li J, Ao J-P, Jiang S-H, Kong F-Z, Tu L, Zhuang C, et al. Silencing of WISP3 suppresses gastric cancer cell proliferation and metastasis and inhibits Wnt/ $\beta$-catenin signaling. Int J Clin Exp Pathol. 2014;7:6447-61.

17. Li-Chang HH, Kasaian K, Ng Y, Lum A, Kong E, Lim H, Jones SJ, Huntsman DG, Schaeffer DF, Yip S. Retrospective review using targeted deep sequencing reveals mutational differences between gastroesophageal junction and gastric carcinomas. BMC Cancer. 2015;15:32.

18. Mafficini A, Amato E, Fassan M, Simbolo M, Antonello D, Vicentini C, Scardoni M, Bersani S, Gottardi $M$, Rusev $B$, et al. Reporting tumor molecular heterogeneity in histopathological diagnosis. PloS one. 2014;9:e104979.

19. Lim D, Kim N-K, Park H-S, Lee S-H, Cho Y-M, Oh SJ, Kim T-H, Kim H. Identification of candidate genes related to bovine marbling using protein-protein interaction networks. International journal of biological sciences 2011, 7.

20. van Schie EH, van Amerongen R. Aberrant WNT/CTNNB1 Signaling as a Therapeutic Target in Human Breast Cancer: Weighing the Evidence. Frontiers in cell developmental biology. 2020;8:25.

21. X X, C QL, L X ZSR, Z Z, X HDPL, Z YG. C S: Inhibition of DYRK1A-EGFR axis by p53-MDM2 cascade mediates the induction of cellular senescence. Cell death disease. 2019;10:282.

22. Kong F, Deng X, Kong X, Du Y, Li L, Zhu H, Wang Y, Xie D, Guha S, Li Z, et al. ZFPM2-AS1, a novel IncRNA, attenuates the $\mathrm{p} 53$ pathway and promotes gastric carcinogenesis by stabilizing MIF. Oncogene. 2018;37:5982-96.

23. Huang S, Li Y, Yuan X, Zhao M, Wang J, Li Y, Li Y, Lin H, Zhang Q, Wang W, et al. The UbL-UBA Ubiquilin4 protein functions as a tumor suppressor in gastric cancer by p53-dependent and $p 53-$ independent regulation of p21. Cell death differentiation. 2019;26:516-30.

\section{Figures}




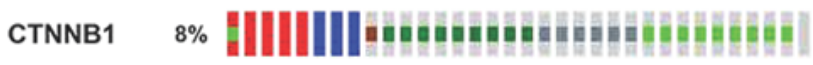

Genetic Alteration

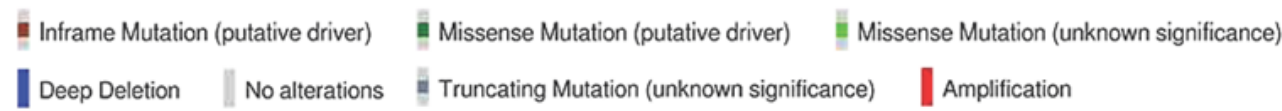

A

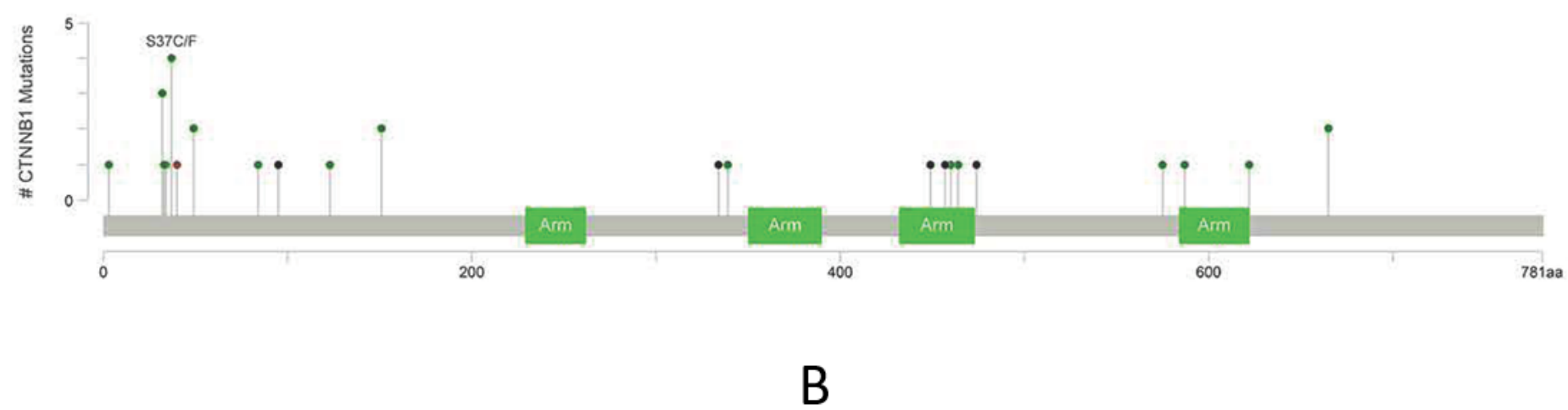

\section{Figure 1}

Mutation frequency (A) and types (B) of CTNNB1 in GC reproduced from the cancer Genome Atlas (TCGA) database. 


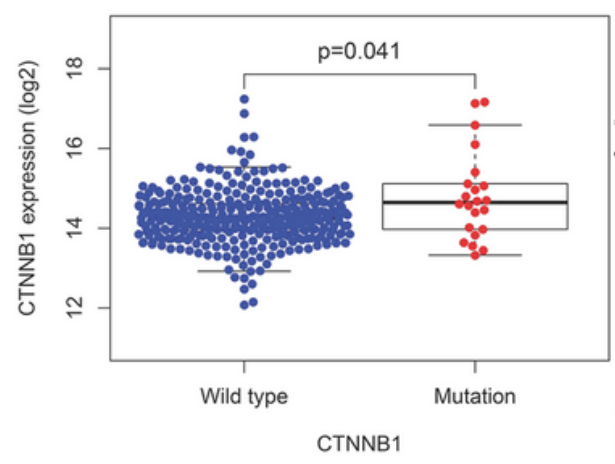

A

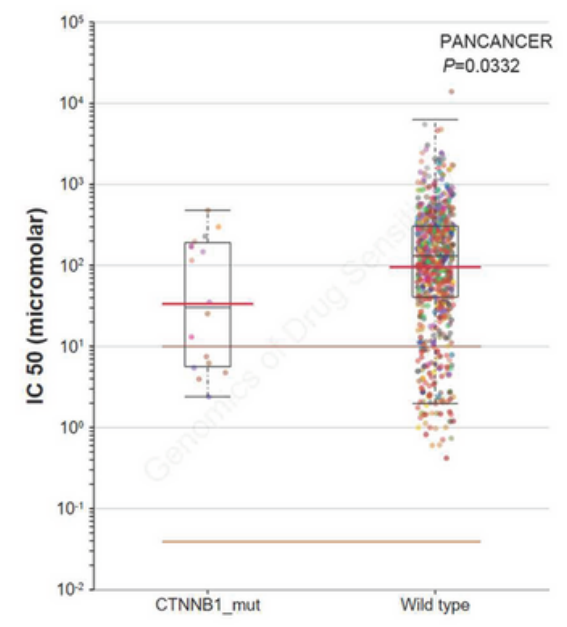

D

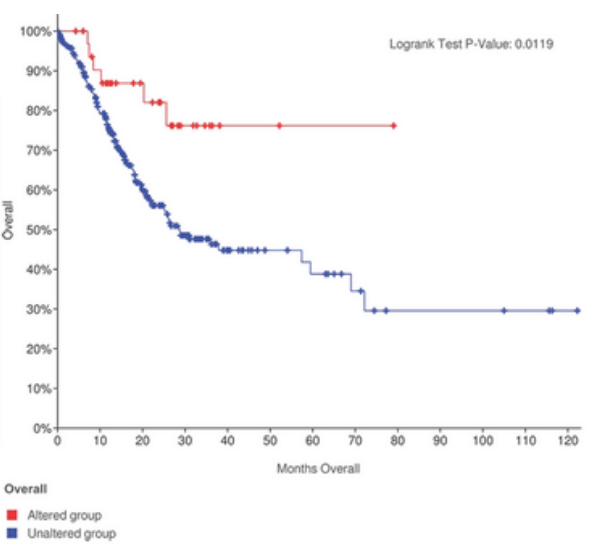

B

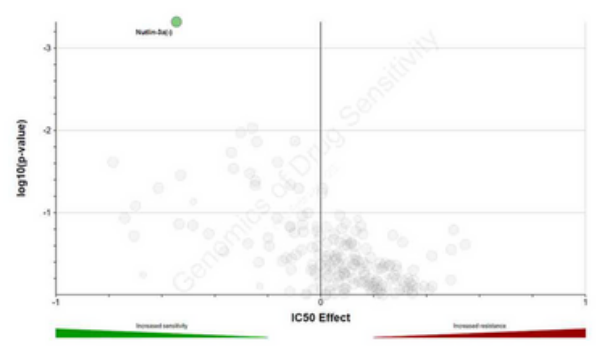

$E$

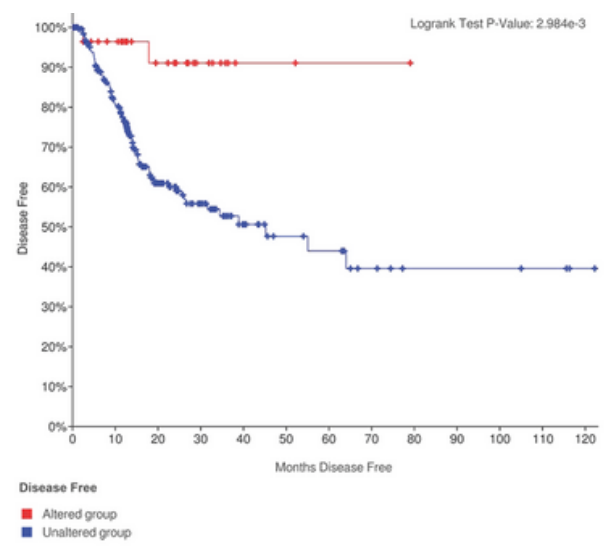

C

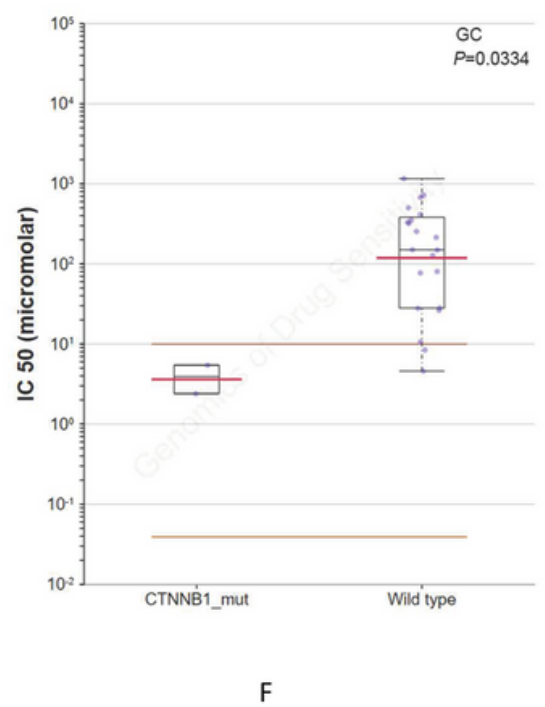

Figure 2

Mutations of CTNNB1 are associated with GC prognosis and drug selection. (A) Correlation between the CTNNB1 mutation and wild type of mRNA expression. (B, C) Kaplan-Meier overall survival and diseasefree survival for GC patients stratified by the CTNNB1 mutation. (D, E) Scattered plot and volcano plot showed that multiple cancer cell types with the CTNNB1 mutation were significantly inhibited by Nutlin3a. (F) Reproduction of the GDSC database by excluding cancer of other types showed that GC cells with the CTNNB1 mutation was also significantly inhibited by Nutlin-3a . 


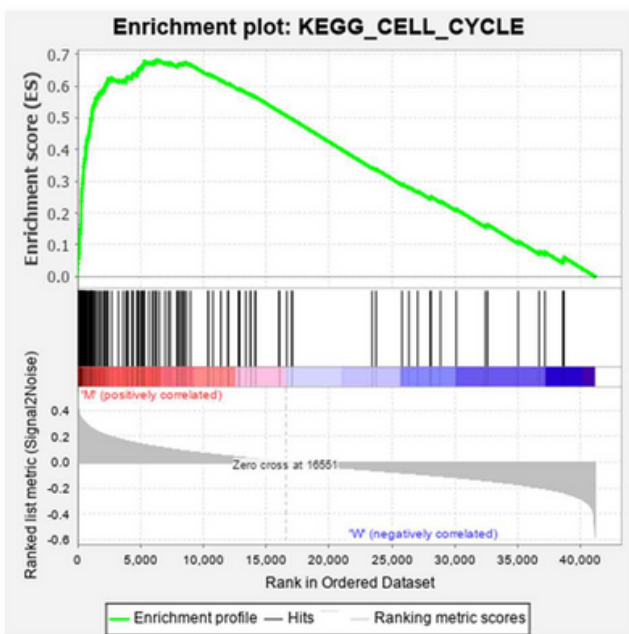

A

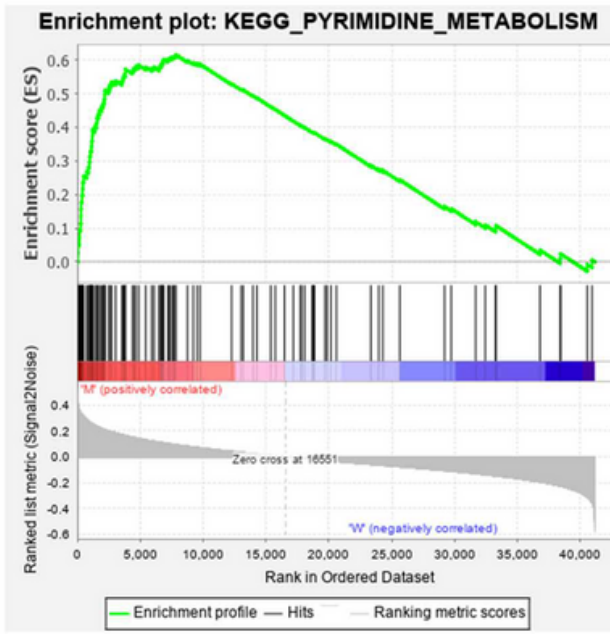

D

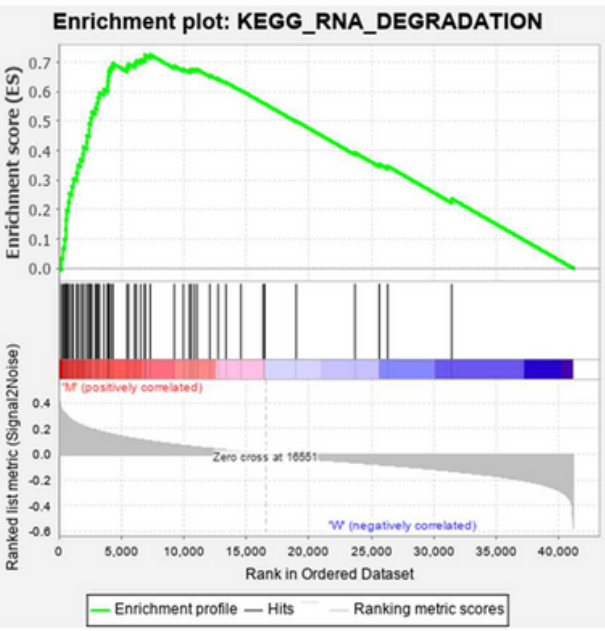

B

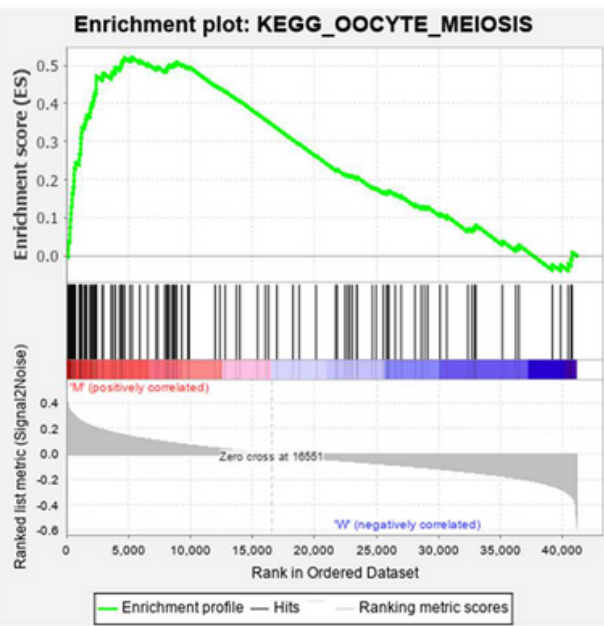

$\mathrm{E}$
Enrichment plot: KEGG_SPLICEOSOME

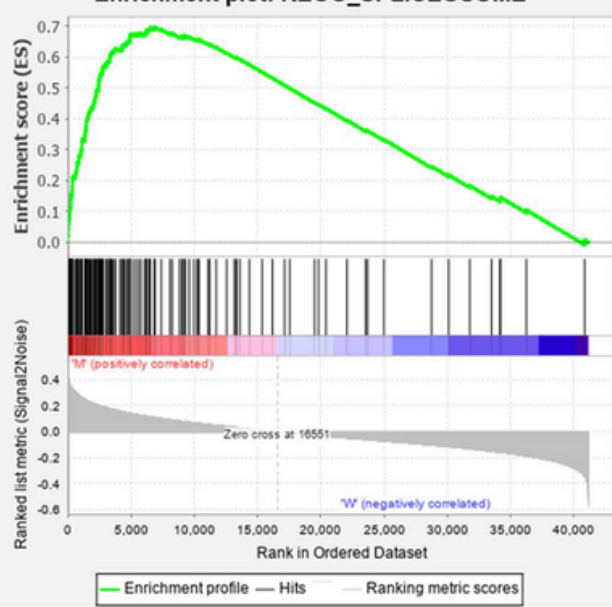

C

Enrichment plot: KEGG_P53_SIGNALING_PATHWAY
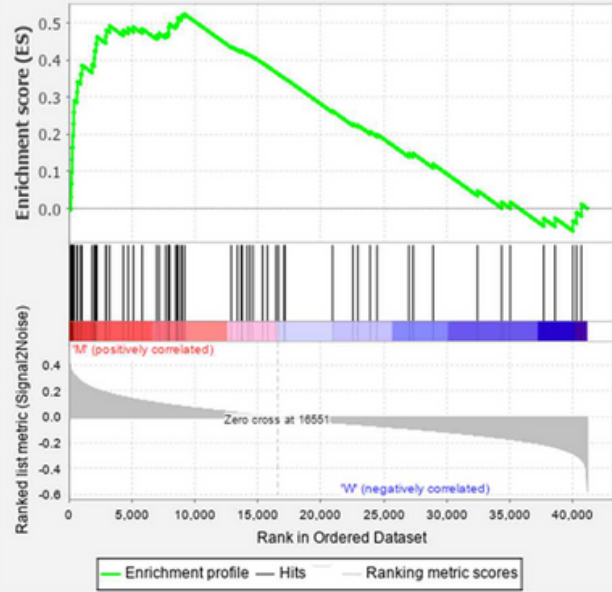

$\mathrm{F}$

\section{Figure 3}

GSEA results of the CTNNB1 mutation in GC patients. 


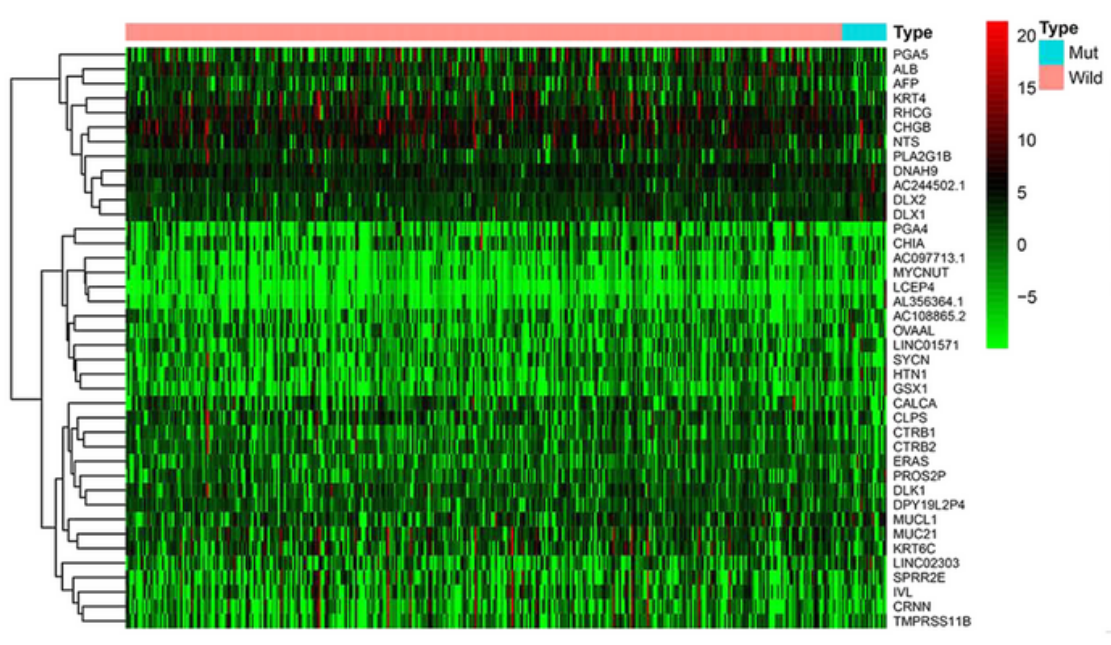

A
Volcano

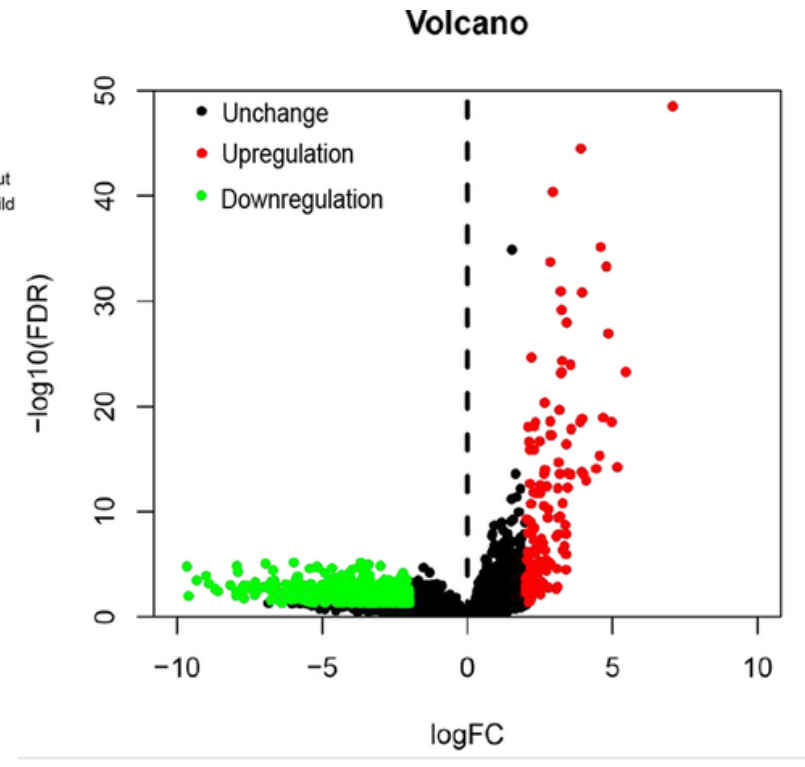

B

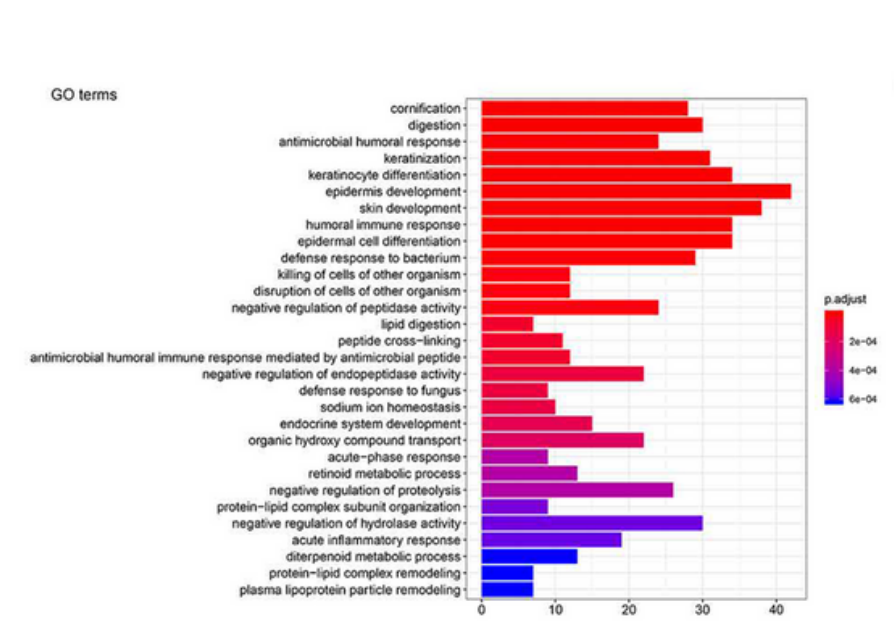

C

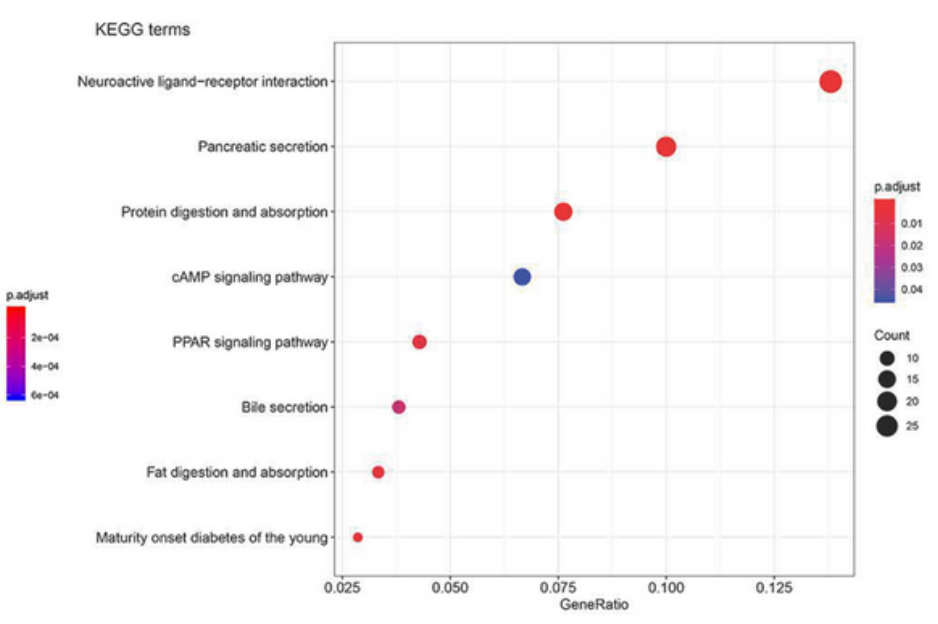

D

\section{Figure 4}

Function enrichment results of differentially expressed genes (DEGs). (A) Heat map of DEGs. (B)Volcano plot for DEGs. (C) The GO enrichment terms of DEGs. (D) The KEGG pathway analysis of DEGs. 


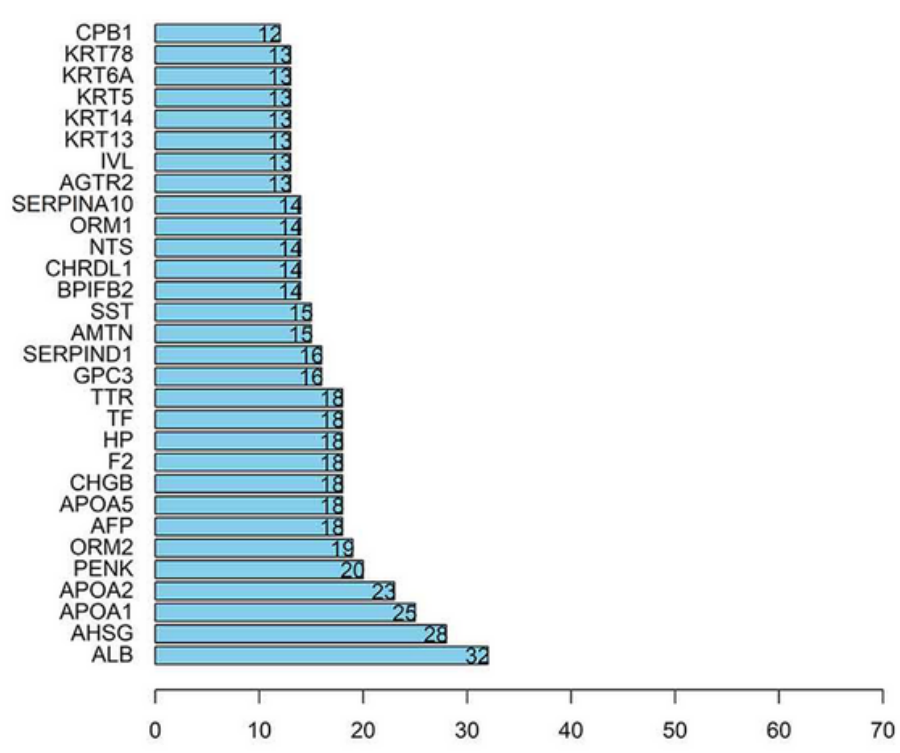

A

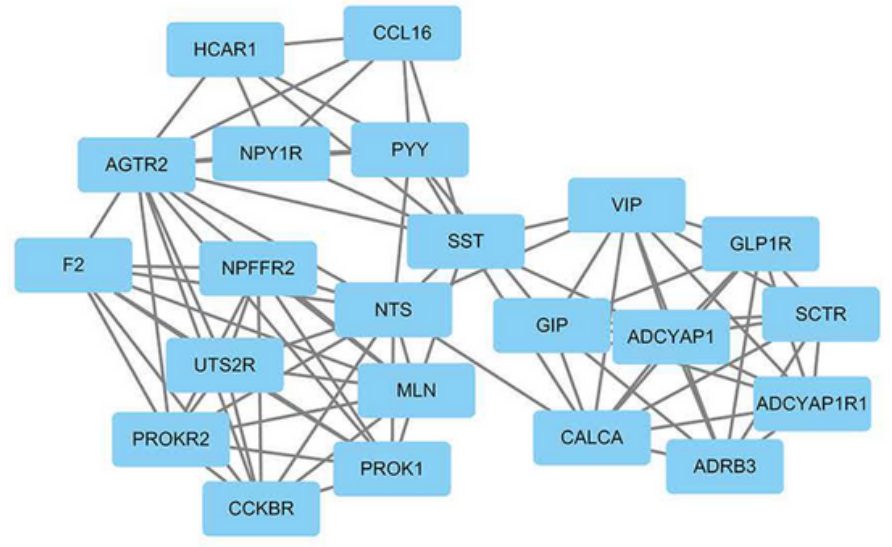

$\mathrm{C}$

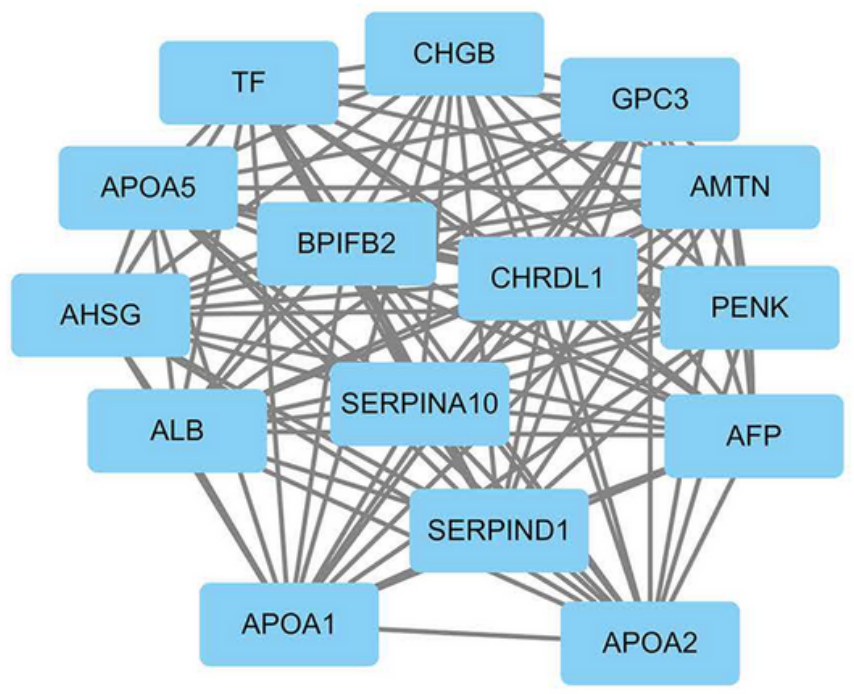

B

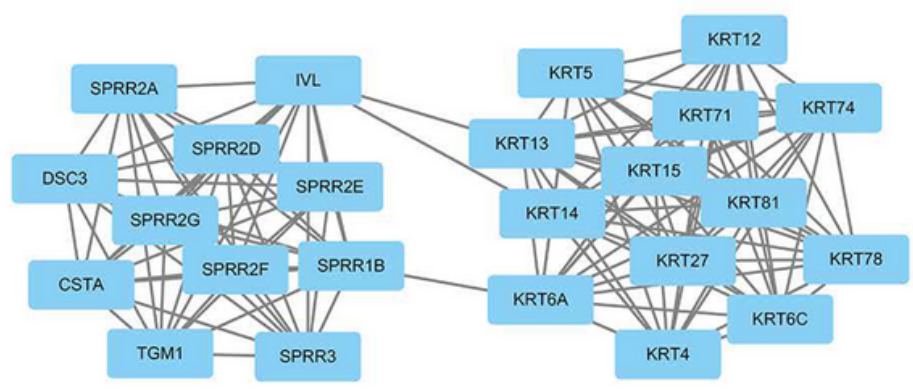

D

\section{Figure 5}

Top three modules from the PPI network. (A) PPI network of module 1. (B) PPI network of module 2. (C) PPI network of module 3 . 